\title{
THE INDUCTION OF CYTOTOXICITY BY PTEROSTILBENE IN VARIOUS HUMAN CANCER CELL LINES
}

\author{
JOANNA WAWSZCZYK*, KATARZYNA JESSE, ALEKSANDRA SZEWERNIAK, \\ MAŁGORZATA KAPRAL and LUDMIŁA WĘGLARZ
}

Department of Biochemistry, Jedności 8, 41-200 Sosnowiec, Poland, School of Pharmacy with the Division of Laboratory Medicine in Sosnowiec, Medical University of Silesia, Katowice, Poland

\begin{abstract}
Pterostilbene is a naturally occurring compound found primarily in blueberries and grapes. It has been found to possess several biological activities such as antioxidative and anti-inflammatory. The study evaluated the cytotoxic activity of pterostilbene in various human cancer cell lines, i.e., melanoma (A2058, C32), colon carcinoma (HT-29, SW1116), breast adenocarcinoma (MCF-7, SKBR3) and ovary adenocarcinoma (SKOV3). The cells were treated with pterostilbene concentrations ranging from 5 to $75 \mu \mathrm{M}$ for $72 \mathrm{~h}$. The cytotoxicity of pterostilbene was evaluated using the Sulforhodamine B assay and expressed as a percentage of that of untreated control cells. The concentration of pterostilbene required for $50 \%$ reduction of cell viability $\left(\mathrm{IC}_{50}\right)$ was calculated from log dose-response curves. The results of this study showed that pterostilbene exerted dosedependent cytotoxic effect on cancer cells. Among the all cell lines tested, the C32 melanoma cells were the most sensitive to the cytotoxic effect of pterostilbene $\left(\mathrm{IC}_{50} \sim 10 \mu \mathrm{M}\right)$ and $\mathrm{SW} 1116$ colon cancer cells showed the lowest sensitivity $\left(\mathrm{IC}_{50} \sim 70 \mu \mathrm{M}\right)$.
\end{abstract}

Keywords: pterostilbene, cytotoxic activity, SRB assay, cancer cell lines

Cancer is one of the major public health problems all over the world, as it is one of the leading causes of death worldwide $(1,2)$. Epidemiological studies have consistently shown that consumption of a diet including fruits and vegetables is strongly associated with reduced risk of cancer (3). A large number of plants and their isolated constituents have been shown to possess potential anticancer activity (4). Stilbenes are phytochemicals present in berries, grapes, peanuts, and red wine. Recently, these compounds have attracted increasing attention and interest due to their wide range of healthbeneficial effects (5). A widely studied stilbene, resveratrol, has been shown to exert antioxidant, anti-inflammatory, chemopreventive and antiaging effects in many biological systems. Resveratrol is potentially capable of inhibiting carcinogenesis at the stages of initiation, promotion and progression (6). Pterostilbene (trans-3,5dimethoxy-4'-hydroxystilbene) is a natural analog of resveratrol showing higher bioavailability and longer half-life in vivo than the latter (7) which makes it a promising dietary factor for chemopre- vention (8). Due to its close structural similarity to resveratrol pterostilbene possesses resveratrol-like health benefits (9). Recent studies showed that pterostilbene exhibited the hallmark characteristics of a valuable anticancer agent including modulation of expression of phase II detoxifying enzymes, regulation of aberrant cell cycle or induction of cell death $(10,11,12)$. Despite the reports on the biological activity of pterostilbene, data on its cytotoxicity against cancer cells are still limited. Therefore, the main goal of this study was to investigate the cytotoxicity of pterostilbene in human cancer cells of different origin, i.e., tumor cells derived from colon, breast and ovary as well as malignant melanoma cells and to compare their sensitivity to pterostilbene. Various cell lines may differ in their sensitivity towards this stilbene, so the use of a broader spectrum of cell lines was considered to ensure better insight into its cytotoxic activity. In addition, the study aimed at evaluating whether the sensitivity of breast and ovarian cancer cells to pterostilbene could be correlated to their ER and HER2 status.






\section{EXPERIMENTAL}

\section{Cell lines and culture conditions}

The seven selected cancer cell lines used in this research were derived from human: ovary adenocarcinoma (SKOV3, ATCC No. HTB-77) breast adenocarcinoma (SKBR3 ATCC No. HTB-30; MCF-7 ATCC No. HTB-22) colon carcinoma (HT-29 ATCC No. HTB-38; SW1116 ATTC No. CCL-233) and melanoma (A2058 ATCC No. CRL-11147; C32 ATCC No. CRL-1585). All cell lines were obtained from LGC Promochem (Lomianki, Poland). The five cancer cell lines were cultured in MEM medium (Sigma Aldrich) supplemented with 10\% fetal bovine serum (PanBiotech), $100 \mathrm{U} / \mathrm{mL}$ penicillin and 100 $\mu \mathrm{g} / \mathrm{mL}$ streptomycin (Sigma Aldrich), while the two cell lines (SKBR3 and SKOV3) were cultured in McCoy's medium (Sigma Aldrich) containing 10\% fetal bovine serum (PanBiotech), $100 \mathrm{U} / \mathrm{mL}$ penicillin and $100 \mu \mathrm{g} / \mathrm{mL}$ streptomycin (Sigma Aldrich). The cell cultures were cultivated as monolayers at $37^{\circ} \mathrm{C}$ in a humidified atmosphere containing $5 \% \mathrm{CO}_{2}$.

\section{Preparation of pterostilbene stock solution}

Pterostilbene was purchased from Sigma Aldrich (Cat. number P1499, purity $=97 \%$ ). The stock solution of pterostilbene was prepared in dimethyl sulfoxide (DMSO) and further diluted in sterile culture medium to desired concentrations immediately before use. The final DMSO concentration in the working solutions was $0.1 \%$.

\section{Cytotoxicity assay}

The cytotoxic effect of pterostilbene on cancer cells was analyzed by using an in vitro toxicology assay kit, which is Sulforhodamine B (SRB) based (Sigma Aldrich). The SRB is a dye that binds electrostatically to cellular proteins under mild acidic conditions. The amount of dye incorporated into protein and extracted under basic conditions was measured colorimetrically. The absorbance value is related proportionally to the total biomass and consequently, cell number (13). Cells were seeded in 96-well plates at an initial density of 2000-5 000 cells (depending upon the cell line) in $200 \mu \mathrm{L}$ of culture medium and allowed to adhere and grow for 24 $\mathrm{h}$. The medium was then removed from each well and replaced with fresh medium containing pterostilbene $(5,10,20,40,50,60,75 \mu \mathrm{M})$ and the cells were cultured for $72 \mathrm{~h}$. After removal of culture media from the wells, the cells were washed with phosphate buffered saline (PBS) and fixed in 10\% trichloroacetic acid, followed by 5 washes with deionized water. Cells were stained with $0.4 \%$ SRB for $30 \mathrm{~min}$. Afterwards, plates were washed with $1 \%$ acetic acid and air-dried. After the liberation of incorporated SRB with $10 \mathrm{mM}$ Tris- $\mathrm{HCl}$, absorbance was measured at $570 \mathrm{~nm}$ and $690 \mathrm{~nm}$ (reference wavelength) using the multiplate reader Labtech LT-5000. The viability of treated cells was expressed as a percentage of untreated control cells. The drug concentration that reduced the viability of cells by $50 \%\left(\mathrm{IC}_{50}\right)$ compared to untreated controls was determined by fitting a four-parameter logistic model (Hill equation) using computer curve-fitting software (GraphPad Prism ver. 7, San Diego, USA) to the experimental data.

\section{Statistical analysis}

Statistical analysis was performed with the use of Statistica PL ver. 12.0 Software (StatSoft). The examined parameters were first evaluated for normal distribution (Shapiro-Wilk test). One-way analysis of variance (ANOVA) with Tukey's post$h o c$ test was used to evaluate significances between examined groups. Results were expressed as the means \pm standard deviation (SD). Differences with a probability (p) value less than 0.05 were considered statistically significant.

\section{RESULTS}

Seven different human cancer cell lines were used to screen for the cytotoxic activity of pterostilbene in vitro. Melanoma (A2058, C32), colon carcinoma (HT-29, SW1116), breast adenocarcinoma (MCF-7, SKBR3) and ovary adenocarcinoma (SKOV3) cells were treated with increasing concentrations of pterostilbene $(5-75 \mu \mathrm{M})$ for $72 \mathrm{~h}$ and viable cells were detected with SRB assay. Pterostilbene reduced cell viability in all cell lines tested here and in a concentration-dependent fashion (Fig. 1). In addition, Figure 1 shows the respective $\mathrm{IC}_{50}$ value for pterostilbene in each cell line, calculated from these concentration-response curves.

The cytotoxic effect of pterostilbene on HT-29 and SW1116 colon carcinoma cells is shown in Figures $1 \mathrm{~A}$ and $1 \mathrm{~B}$. At a concentration of $5 \mu \mathrm{M}$, it did not affect colon carcinoma cell viability. A significant viability suppression of both cell lines was observed at higher concentrations $(\geq 10 \mu \mathrm{M})$ of pterostilbene. Furthermore, the much stronger inhibitory effect was observed in HT-29 cells than SW1116 cells. At 75 $\mu \mathrm{M}$ pterostilbene, the viability of HT-29 and SW1116 cells was reduced by $95.4 \%$ and $50 \%$, respectively. The $\mathrm{IC}_{50}$ values of about $20 \mu \mathrm{M}$ and $70 \mu \mathrm{M}$ for HT-29 and SW1116 cells respectively, reflected their different sensitivity to pterostilbene. 
Colon carcinoma cell line
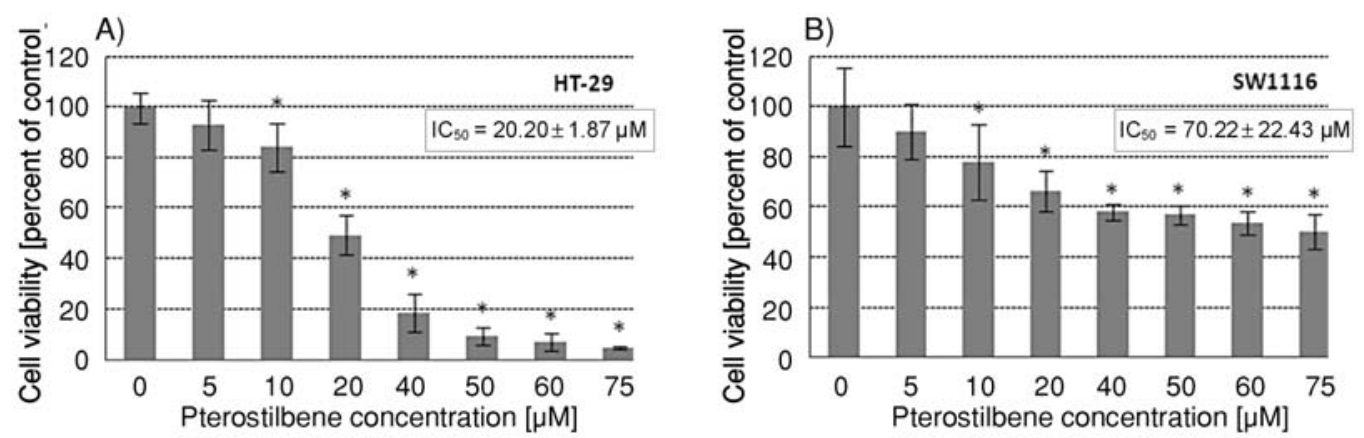

Melanoma cell line
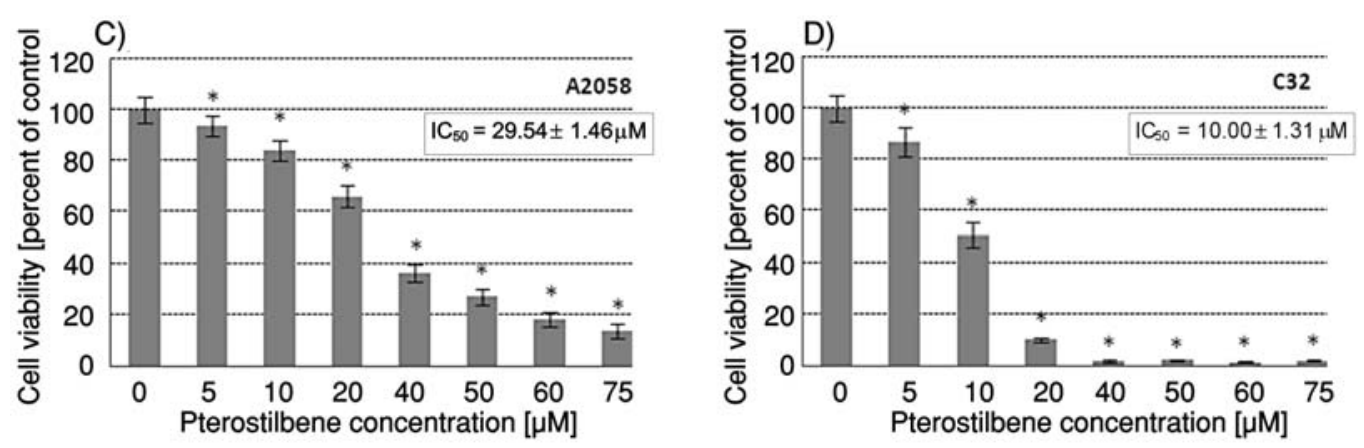

Breast cancer cell line
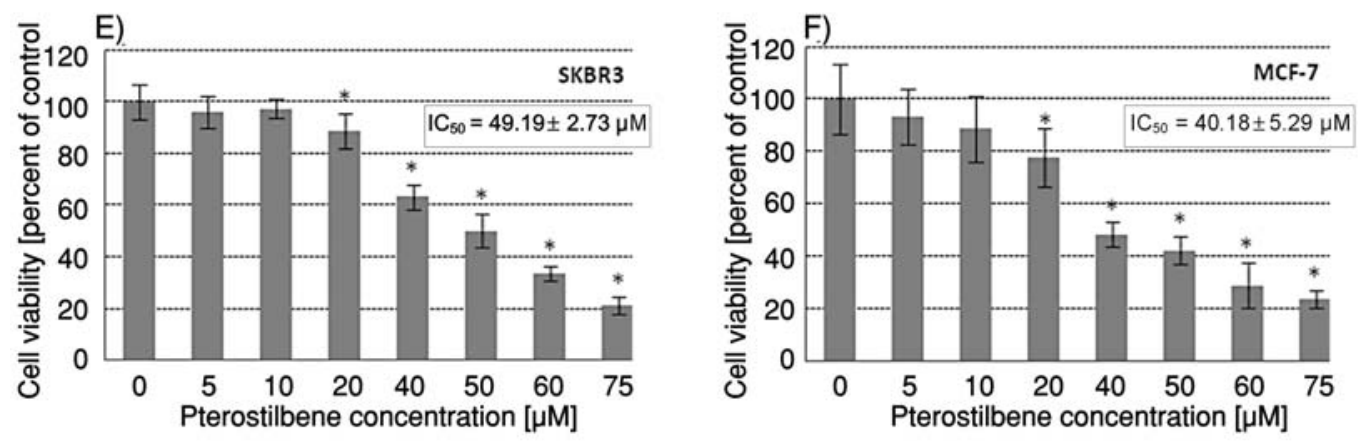

Ovarian cancer cell line



Figure 1. Cytotoxic effect of pterostilbene on human cancer cells after $72 \mathrm{~h}$ treatment. The results are expressed as percentage of untreated control (the means $\pm \mathrm{SD} ; * \mathrm{p}<0.05$ vs. control) 
The A2058 and C32 cell lines were used as an experimental in vitro model of skin melanoma malignum. These cell lines originate from different melanoma types: melanotic (A2058) and amelanotic (C32). Pterostilbene at the all concentrations used (5$75 \mu \mathrm{M})$ significantly suppressed the viability of both A2058 (Fig. 1C) and C32 (Fig. 1D) cells in a concentration-dependent manner. Amelanotic C32 cells were more sensitive to pterostilbene than melanotic A2058 cells, showing above $80 \%$ inhibition at relatively low concentration of $20 \mu \mathrm{M}$ pterostilbene, whereas in A2058 cell cultures comparable cytotoxicity was achieved with its higher concentrations (60 and $75 \mu \mathrm{M})$. The $\mathrm{IC}_{50}$ value for pterostilbene was almost 3-fold higher in A2058 cells than in C32 cells.

Cytotoxicity of pterostilbene in SKBR3 and $\mathrm{MCF}-7$ adenocarcinoma cells is demonstrated in Figures $1 \mathrm{E}$ and $1 \mathrm{~F}$. Pterostilbene at the concentrations up to $10 \mu \mathrm{M}$ did not affect SKBR3 and MCF-7 cell viability. A substantial cell viability reduction was observed in cells incubated with higher concentrations $(\geq 20 \mu \mathrm{M})$. The maximum decrease of SKBR3 and MCF-7 cell viability versus corresponding control cultures $(78.6 \%$ and $76.5 \%$ respectively) was evoked by the highest dose of pterostilbene. For SKBR3 cell line, the $\mathrm{IC}_{50}$ was $49.19 \mu \mathrm{M}$. The comparable value was obtained for MCF-7 cells (40.18 $\mu \mathrm{M})$.

The SKOV3 cell line was used as an experimental in vitro model of ovary adenocarcinoma. The experimental data presented in Figure $1 \mathrm{G}$ indicate that the exposure of SKOV3 cells to pterostilbene exhibited a dual effect. Pterostilbene at a concentration of $5 \mathrm{mM}$ induced an increase in cell viability, while at higher concentrations $(\geq 40 \mu \mathrm{M})$ it was cytotoxic. The most pronounced reduction of cell viability by pterostilbene was observed in cell cultures treated with $75 \mu \mathrm{M}$ of pterostilbene. The $\mathrm{IC}_{50}$ for stilbene was found to be $47.03 \mu \mathrm{M}$.

\section{DISCUSSION}

Pterostilbene is a natural methoxylated resveratrol derivative. It has higher biostability due to slower metabolism and lower excretion rate in comparison to other stilbenes, which renders it a better potential health-promoting nutraceutical (14). Recent studies have shown that pterostilbene could be a novel promising chemopreventive and chemotherapeutic agent (15). The mechanisms underlying its anticancer activity have not been fully elucidated and are believed to include e.g. anti-oxidative, anti-proliferative and cytotoxic effects. The antioxidant properties of pterostilbene are assumed to enable this com- pound to protect cells from oxidative damage. Pterostilbene may exhibit cancer cell death via apoptosis and autophagy and apoptosis rather is postulated as the main mechanism of its cytotoxic activity $(10,16,17)$. Prooxidant properties of pterostilbene, particularly at higher concentrations, are held likely to be responsible for its pro-apoptotic effects. Pterostilbene has been shown to induce the intrinsic apoptotic pathway in cancer cells by increasing intracellular ROS production, mitochondrial depolarization, regulation of mitochondrial proteins Bcl-2, Bax, cytochrome c and caspase cascade activation. Proapoptotic mechanism of pterostilbene also may include extrinsic pathway (10, 18). Moreover, pterostilbene-induced cell death may also occur via the caspase-independent mechanism. Recent studies revealed that pterostilbene induced lysosomal membrane permeabilization leading to the activation of caspase-independent lysosomal cell death program (19). Other studies have shown the cytotoxic and growth inhibitory effect of pterostilbene on cancer cells such as lymphoma (18), liver (20), lung (21) and glioma (22) cancer cells. The present experiments were aimed at comparing the cytotoxic activity of pterostilbene among several types of human cancer cell lines. The concentration range of 5 to 75 $\mu \mathrm{M}$ was selected for testing based on a report of plasma levels of pterostilbene after iv administration in mice wherein plasma levels of pterostilbene were observed to vary from 1 to $116 \mu \mathrm{M}$ over the range of $5 \mathrm{~min}$ to $480 \mathrm{~min}$ (23). Detectable concentrations of pterostilbene and its glucuronidated metabolite $(0.05-100 \mu \mathrm{g} / \mathrm{mL})$ were also detected in rat's serum up to six hours after oral consumption (24). It was also reported that the levels of pterostilbene consumed with the diet exceeded $20 \mu \mathrm{M}$ in mice colonic mucosa (25). Only a few studies evaluated the safety of pterostilbene administration to humans thus far (14), but to our knowledge, there is no report on serum levels of pterostilbene in humans. Currently, it is not known whether oral consumption of pterostilbene might be sufficient to elicit its levels commensurate with cancer chemopreventive efficiency. However, it is important to note that metabolites of pterostilbene may also contribute to biological effects of this stilbene (25).

Pterostilbene reduced cell viability in all tested cell lines in a concentration-dependent manner. Cells from different origins showed different sensitivities to pterostilbene. The statistically important decrease of cell viability in melanoma cells (C32 and A2058) was evoked by pterostilbene at concentration $5 \mu \mathrm{M}$ whereas in ovarian cancer cells (SKOV3) by $40 \mu \mathrm{M}$. 
Since pterostilbene may easily be included in the human diet, its potential activity against human colon cancer cells is worth closer investigation. The current study demonstrates the cytotoxic activity of pterostilbene (at concentrations $=10 \mu \mathrm{M}$ ) on two colon cancer cell lines with $\mathrm{IC}_{50}$ values of $20.20 \mu \mathrm{M}$ (HT-29) and 70.22 $\mu \mathrm{M}$ (SW1116). Other literature has confirmed the cytotoxic activity of pterostilbene on colon cancer cells HT-29 $\left(\mathrm{IC}_{50}=23.8 \mu \mathrm{M}\right)$ after $72 \mathrm{~h}$ treatment (26). The weaker cytotoxic effect of pterostilbene on HT-29 cells (higher $\mathrm{IC}_{50}$ values) was observed by Mena et al. (19) and Sun et al. (25) which could likely be due to a shorter incubation period of treatment $(48 \mathrm{~h})$. Studies carried out by Harun and Ghazali (27) did not support the cytotoxic effect of pterostilbene at concentrations up to 100 $\mu \mathrm{M}$ against HT-29 cells after 24 treatment. Pterostilbene was also reported to reduce the viability of other colon cancer cell lines such as HCT1116 (25) and Caco-2 (26). As observed in this study, a more pronounced cytotoxic effect of pterostilbene was observed against HT-29 cells presenting low expression of enzyme cyclooxygenase-2 (COX-2) than against SW1116 cells with its high expression. COX-2 plays a crucial role in the inflammation process, cell proliferation and colon carcinogenesis (28). Since Chiou et al. (11) reported marked decrease of azoxymethane-induced COX-2 expression in mice following dietary pterostilbene intake, determination of the possible COX-2 pathway involvement in cytotoxic activity of pterostilbene in SW1116 and HT-29 cells is warranted.

Thus far, limited studies have investigated cytotoxic and antitumor effects of pterostilbene on skin cancer including melanoma (21). The current study showed that pterostilbene at all concentrations exerted cytotoxic activity against both amelanotic and melanotic melanoma cells after $72 \mathrm{~h}$ treatment. The most pronounced effect was exerted by pterostilbene against the $\mathrm{C} 32$ amelanotic melanoma cells. The strongest cytotoxicity was observed with 40 to $75 \mu \mathrm{M}$ of pterostilbene and $\mathrm{IC}_{50}$ for $\mathrm{C} 32$ cell line was $10 \mu \mathrm{M}$ and it was 2.9-fold lower than that for A2058 cell line. Other research supports cytotoxic effects of this stilbene against highly invasive SK-MEL-2 and MeWo melanoma cells and over a wide range of concentrations (10-100 $\mu \mathrm{M})$ (29). On the other hand, Benlloch at al. (23) reported that pterostilbene at low concentrations $(1-5 \mu \mathrm{M})$ does not alter melanoma cell growth and viability after $72 \mathrm{~h}$.

In the present experiment, the cytotoxic effect of pterostilbene on breast and ovarian cancer cells with different expression of estrogen receptor (ER) and human epidermal growth factor receptor 2 (HER2) was also evaluated. ER and HER2 play pivotal role in those cancer cells and their expression is an important factor in determining the therapy effectiveness of breast and ovarian cancer $(30,31,32)$. Pterostilbene significantly decreased viability of MCF-7 (ER ${ }^{+} / \mathrm{HER}^{-}$; responsive to estradiol) and SKBR3 (ER ${ }^{-} / \mathrm{HER}^{+}{ }^{+}$; nonresponsive to estradiol) breast cancer cells as well as SKOV3 (ER ${ }^{-} / \mathrm{HER}^{+}{ }^{+}$, nonresponsive to estradiol) ovarian cancer cells. The obtained experimental $\mathrm{IC}_{50}$ values for all these cells were similar, thus, the cytotoxic effect of pterostilbene appears not to be associated with their ER/HER2 status. These data concur with the results published by Alosi et al. (33) who demonstrated that pterostilbene (20-100 $\mu \mathrm{M})$ induced a significant concentration and timedependent growth decrease of MDA-MB-231 and MCF-7 breast cancer cells. In the other studies, pterostilbene offered cytotoxic effect against SKOV3 cells at a concentration of $\mathrm{IC}_{50}$ about $55 \mu \mathrm{M}$ (34).

It is worth mentioning that pterostilbene exhibited a dual effect on the viability of SKOV3 cells. At a lower concentration $(5 \mu \mathrm{M})$ it stimulated while at higher concentration $(\geq 40 \mu \mathrm{M})$ it reduced SKOV3 cell viability. A similar biphasic activity of pterostilbene was also observed towards primary macrophages (35). This biphasic dose-response model (characterized by a low-dose stimulation and a highdose inhibition) on human tumor cell lines has also been demonstrated for other stilbenes: resveratrol (36, 37) and piceatannol (38). Based on those reports it is important to perform more detailed studies of biological activity of stilbenes in a wide range of concentration.

The present results indicate that pterostilbene may be a potential anti-cancer agent because it decreases the viability of all tested colon cancer cell lines. Melanoma C32 cells, followed by colon carcinoma HT-29 cells and melanoma A2058 were the most sensitive cells to pterostilbene, with $\mathrm{IC}_{50}$ values lower than $30 \mu \mathrm{M}$, while colon carcinoma SW1116 cells were the least sensitive. Although we did not determine whether it was selectively cytotoxic for neoplastic cells, Sun et al. (25) demonstrated that the growth of normal human colon fibroblasts was not altered following 24 and 48-hour exposure to lower concentrations $(5-40 \mu \mathrm{M})$ of pterostilbene. This result is also consistent with the findings of Dewi et al. (39) who did not observe the antiproliferative activity of pterostilbene $(12.5-100 \mu \mathrm{M})$ against normal myofibroblasts and skin fibroblasts.

In conclusion, the present results showed the cytotoxic effect of pterostilbene against cancer cells derived from four of the most prevalent cancers: colon, melanoma, breast and ovary cancer. It can 
also be concluded that cytotoxicity induced by this stilbene in breast and ovarian cancer cells is not associated with their ER and HER2 status. However, further studies must be done to seek for the molecular mechanisms of its pterostilbene activity.

\section{Acknowledgments}

This work was supported by grants no. KNW$2-013 / \mathrm{N} / 5 / \mathrm{K}$ and $\mathrm{KNW}-2-\mathrm{I} 18 / \mathrm{D} / 6 / \mathrm{N}$ from the Medical University of Silesia (Katowice, Poland).

\section{REFERENCES}

1. Parasramka M.A., Gupta S.V.: J. Oncol. 2012, 709739 (2012).

2. Berghe W.V.: Pharmacol. Res. 65, 565 (2012).

3. Turati F., Rossi M., Pelucchi C., Levi F., La Vecchia C.: Br. J. Nutr. 113, 102 (2015).

4. Elsayed E.A., Sharaf-Eldin M.A., Wadaan M.: Asian Pac. J. Cancer Prev. 16, 4671 (2015).

5. Sirerol J.A., Rodríguez M.L., Mena S., Asensi M.A., Estrela J.M., Ortega A.L.: Oxid Med. Cell. Longev. 2016, 3128951 (2016).

6. Kondratyuk T.P., Park E.J., Marler L.E., Ahn S., Yuan Y. et al.: Mol. Nutr. Food Res. 55, 1249 (2011).

7. Kapetanovic I.M., Muzzio M., Huang Z., Thompson T.N., McCormick D.L.: Cancer Chemother. Pharmacol. 68, 593 (2011).

6. Ferrer P., Asensi M., Segarra R., Ortega A., Benlloch M. et al.: Neoplasia 7, 37 (2005).

9. McCormack D., McFadden D.: Oxid. Med. Cell. Longev. 2013, 575482 (2013).

10. McCormack D., McFadden D.: J. Surg. Res. 173, 553 (2012).

11. Chiou Y.S., Tsai M.L., Nagabhushanam K., Wang Y.J., Wu C.H. et al.: J. Agric. Food Chem. 59, 2725 (2011).

12. Ma Z., Yang Y., Di S., Feng X., Liu D. et al.: Sci. Rep. 7, 8091 (2017).

13. Skehan P., Storeng R., Scudiero D., Monks A., McMahon J. et al.: J. Natl. Cancer Inst. 82, 1107 (1990).

14. Riche D.M., McEwen C.L., Riche K.D., Sherman J.J., Wofford M.R. et al.: J. Toxicol. 2013, 463595 (2013).

15. Lee H., Kim Y., Jeong J.H., Ryu J.H., Kim W.Y.: PLoS One 11, e0162335 (2016).

16. Wang Y., Ding L., Wang X., Zhang J., Han W. et al.: Am. J. Transl. Res. 4, 44 (2012).

17. Chen R.J., Ho C.T., Wang Y.J.: Mol. Nutr. Food Res. 54, 1819 (2010).
18. Kong Y., Chen G., Xu Z., Yang G., Li B. et al.: Sci. Rep. 6, 37417 (2016).

19. Mena S., Rodríguez M.L., Ponsoda X., Estrela J.M., Jäättela M., Ortega A.L.: PLoS One 7, e44524 (2012).

20. Guo L., Tan K., Wang H., Zhang X.: Oncol Rep 36, 3233 (2016).

21. Wang Y.J., Lin J.F., Cheng L.H., Chang W.T., Kao Y.H. et al.: J. Hematol. Oncol. 10, 72 (2017).

22. Zielińska-Przyjemska M., Kaczmarek M., Krajka-Kuźniak V., Łuczak M., BaerDubowska W.: Toxicol. In Vitro 43, 69 (2017).

23. Benlloch M., Obrador E., Valles S.L., Rodriguez M.L., Sirerol J.A. et al.: Antioxid. Redox Signal. 24, 974 (2016).

24. Remsberg C.M., Yáñez J.A., Ohgami Y., VegaVilla K.R., Rimando A.M., Davies N.M.: Phytother. Res. 22, 169 (2008).

25. Sun Y., Wu X., Cai X., Song M., Zheng J. et al.: Mol. Nutr. Food Res. 60, 1924 (2016).

26. Paul S., Mizuno C.S., Lee H.J., Zheng X., Chajkowisk S. et al.: Eur. J. Med. Chem. 45, 3702 (2010).

27. Harun Z, Ghazali A.R.: Asian Pac. J. Cancer Prev. 12, 6403 (2012).

28. Brown J.R., DuBois R.N.: J. Clin. Oncol. 23, 2840 (2005).

29. Schneider J.G., Alosi J.A., McDonald D.E., McFadden D.W.: Am. J. Surg. 198, 679 (2009).

30. Neve R.M., Chin K., Fridlyand J., Yeh J., Baehner F.L.: Cancer Cell. 10, 515 (2006).

31. Siddiqa A., Long L.M., Li L., Marciniak R.A., Kazhdan I.: BMC Cancer 8, 129 (2008).

32. Giacinti L., Giacinti C., Gabellini C., Rizzuto E., Lopez M., Giordano A.: J. Cell Physiol. 227, 3426 (2012).

33. Alosi J.A., McDonald D.E., Schneider J.S., Privette A.R., McFadden D.W.: J. Surg. Res. 161, 195 (2010).

34. Dong J., Guo H., Chen Y.: Eur. J. Gynaecol. Oncol. 37, 342 (2016).

35. Adiabouah Achy-Brou C.A., Billack B.: Drug Chem. Toxicol. 40, 36 (2017).

36. Calabrese E.J.: Hum. Exp. Toxicol. 29, 977 (2010).

37. Calabrese E.J., Mattson M.P., Calabrese V.: Hum. Exp. Toxicol. 29, 980 (2010).

38. Vo N.T., Madlener S., Bago-Horvath Z., Herbacek I., Stark N. et al.: Carcinogenesis 31, 2074 (2010).

39. Dewi N.I., Yagasaki K., Miura Y.: Cytotechno$\operatorname{logy} 67,671$ (2015).

Received: 28.07. 2017 